Check for updates

Cite this: Mater. Adv., 2021, 2, 4029

Received 22nd April 2021 Accepted 4th May 2021

DOI: $10.1039 / \mathrm{d} 1 \mathrm{ma} 00369 \mathrm{k}$

rsc.li/materials-advances

\title{
Tunable and ordered porous carbons with folding-like nanoscale framework via interdigitation and twisting $\dagger$
}

\begin{abstract}
Shiori Kubo (D)
Nanoscale folded structures, such as protein folds, extensively exist in nature and are key to controlling various physicochemical functions; however, their controlled expressions in both organic and inorganic solid matter are still limited. In this paper, the sugar ( $D$ - and L-fructose)-derived, hydrothermal synthesis of ordered porous carbonaceous materials with a long-range, folded nanoscale framework is reported. The block copolymer-templated carbonaceous precipitates demonstrate the presence of twisted lamellae-like ordered nanostructural motifs with a repeating distance of ca. $20 \mathrm{~nm}$, as revealed via detailed electron microscopy studies. Furthermore, the synchrotron X-ray scattering study unravels a threefold symmetry, indicating the nanostructural configuration, where the formed mutually nearly identical layering modes are related by a threefold axis and further interpenetrate each other. Owing to this interpenetration, the developed carbonaceous framework is interdigitated on the nanoscale, while the accompanying twisting event folds (i.e., integrates) the system. The proposed mechanism is based on the interplay of the chemical condensation of sugar (i.e., a chiral carbon precursor) and its binding to polymer template inducing layered dimensional ordering. Template removal yields porous carbonaceous bodies with folded lamellae-like ordering modes interpenetrating each other and, therefore, being stabilized, and with the knot-like motif observed locally, due to the complex interdigitation. The extended process controllability of the hydrothermal carbonization allows pore size control by varying the composition of the starting solution. The presented novel porous carbonaceous frameworks would provide large surface area porous bodies with added functions, such as increased intactness (i.e., stability) of the framework, chiral selectivity, or potentially mechanical flexibility, providing interesting features that can be studied for future advancements in electrochemistry and separation and catalysis sciences.
\end{abstract}

\section{Introduction}

Folding or knotting is an important structural attribute of nature that is observed in its various forms and length scales. ${ }^{1,2}$ This functional geometry of matter extensively exists in aqueous milieu of living systems, typically at the nanoscale, with the associated handedness of twisting or symmetry, as represented by protein folding ${ }^{2,3}$ or helix formation of the DNA. ${ }^{1}$ The determined structures are believed to contribute towards dictating various physiological properties of the assemblies by controlling their interactions with the external environment. This includes dynamic control of accessibility towards the internal active sites (e.g., via size/shape selectivity), chemical or thermal stabilities, or

National Institute of Advanced Industrial Science and Technology (AIST), 1-1-1, Higashi, Tsukuba, Japan. E-mail: kubo-shiori@aist.go.jp

$\dagger$ Electronic supplementary information (ESI) available: Details of characterization methods and additional characterization data (electron micrographs, small-angle $\mathrm{X}$-ray scattering and X-ray diffraction patterns, and nitrogen sorption isotherms). See DOI: $10.1039 / \mathrm{d} 1 \mathrm{ma} 00369 \mathrm{k}$ electron and mass transfer. High structural complexity offers more information to be unravelled regarding their exact structures, while mathematical calculations predict various geometrical configurations enabled by complex, yet, well-controlled folding and knotting phenomena.,

Achieving these structural attributes in organic or inorganic stable solid matter will enable the nanostructured or porous bodies to exhibit, in addition to size/shape selectivity, important material properties, such as chiral selectivity ${ }^{6-8}$ or opportunities for controlled catalyst loading or electron and mass transport, which are critical for separation and catalysis sciences ${ }^{9-11}$ as well as for electrochemical applications. ${ }^{12-14}$ Additionally, ordered features, with a deviation from a strictly periodic framework due to folding or twisting, would show interesting electron transfer or photonic properties. ${ }^{15,16}$

When, ultimately, coupled with folding-induced controlled framework interdigitation, new material features of the ordered porous bodies, such as mechanical flexibility (e.g., swelling or resistivity to stress, having a degree of freedom of framework 
movement) and the accompanying dynamic pore shape/size alteration, ${ }^{17}$ or enhanced physical and chemical durability due to the framework entanglement is expected to be observed (owing to the increased degree of framework intactness and reduced framework termini features). Furthermore, realizing such complex folded motifs in a solid form would allow an in-depth study of the topological attributes of biological structures. However, the interdigitated or folded features of matter have rarely been addressed in such "hard" materials.

Considerable progress has been made in the strategies to synthesize one dimensional (1D) twisted porous nanostructured materials of silica ${ }^{18}$ and chiral nematic carbon films (2D), ${ }^{6,7}$ and very recently, helical pore alignment around the 1D carbon fibre has been reported. ${ }^{19}$ However, nanostructural knotting or foldingcharacterized as a self-stabilized ordered system made via interdigitation and twisting (or rotation) - has not been addressed in the field of material synthesis. Sophisticated preparation of molecular building blocks and their mutual coordination has led to chiral covalent organic frameworks $(\mathrm{COFs})^{20}$ or interlocked metal organic framework (MOFs) with mechanical flexibility and associated dynamic porous framework alteration behavior. ${ }^{17}$ However, the pore dimension is intrinsically limited to between several A and 1-2 nm, while the framework is electrochemically non-conductive. Therefore, complex, albeit, well-controlled nanostructures, such as those expressed in biological structures (i.e., existing in hydrated forms in water), are yet to be engineered in a permanent solid form.

In this paper, the results of the synthesis and characterization of a new type of carbonaceous nanostructured solid possessing a folded motif of three-dimensionally interdigitated and twisted ordered layered framework have been reported. To this end, the work takes advantage of hydrothermal synthesis ${ }^{21}$ for exploiting flexible intra- and inter-molecular interactions among precursors sugar, water, and structural template, ${ }^{22-24}$ while an enhanced sustainability of the procedure adds another important synthetic advantage. This paper has been divided into different sections, wherein the initial sections explain the synthesis strategy and accompanying detailed characterization of the resulting carbonaceous particles via electron microscopy, synchrotron small-angle $\mathrm{X}$-ray scattering (SSAXS), and equilibrium gas sorption analyses. In the subsequent sections, the mechanistic view for the formation of the resulting complex, yet controlled carbon nanostructures has been discussed. Based on this, further syntheses via sugar enantiomer (for control of structural handedness) and via a varied block copolymer (BCP) concentration (for pore tuning) have been demonstrated. With the idea that the presented unconventional solid form of folding-like nature and its construction strategy can serve as the first step towards providing a series of nanoscale biomimicking solid objects or porous bodies, potential functions and their application opportunities have been further addressed.

\section{Experimental}

\section{Synthesis}

D-Fructose (99.0+\%) and L-fructose (98+\%) were purchased from Wako Chemicals. Pluronic ${ }^{\circledR} \mathrm{P} 123\left(\mathrm{EO}_{20} \mathrm{PO}_{70} \mathrm{EO}_{20}\right.$, average molecular weight of $\sim 5800 \mathrm{Da}$ ) was purchased from Sigma-Aldrich. All chemicals were used without further purification. In a typical synthesis, fructose and P123 were added to distilled water at molar ratios of fructose $/ \mathrm{P} 123 / \mathrm{H}_{2} \mathrm{O}$ of $1 / 0.013 / 83.40$ (for a diluted system) or $1 / 0.052 / 10.84$ (for a concentrated system). For the diluted system, after complete dissolution, the mixture was hydrothermally treated in a stainless autoclave with a glass inlet at $130{ }^{\circ} \mathrm{C}$ for more than $96 \mathrm{~h}$. For the concentrated system, homogenous gel obtained by repeated ultracentrifugation process was hydrothermally treated in the same manner. The product was washed with excess water and ethanol, followed by drying at $65{ }^{\circ} \mathrm{C}$ in air. Post calcination was performed by heat-treating the product at $550{ }^{\circ} \mathrm{C}$ for $2 \mathrm{~h}$ or at $900{ }^{\circ} \mathrm{C}$ for $1 \mathrm{~h}$ in a nitrogen atmosphere.

\section{Material characterization}

Field-emission scanning electron microscopy (FE-SEM) was performed using HITACHI's Ultrahigh Resolution Scanning Electron Microscopes S4700 and SU9000. Transmission electron microscopy (TEM) images were acquired using a TOPCON EM002B equipped with a Gatan CCD camera with an acceleration voltage of $120 \mathrm{kV}$ and JEOL JEM-1400Plus and JEM-1400Flash with an acceleration voltage of $100 \mathrm{kV}$. For the analyses of the polymercarbon composite materials, samples were embedded in the epoxy resin by thermosetting conducted at $60{ }^{\circ} \mathrm{C}$ for $48 \mathrm{~h}$ and sliced with an ultramicrotome diamond knife (Leica Ultracut) for TEM specimen preparation. The CD measurements were performed using a JASCO 820 spectrometer. SSAXS was performed at the BL8S3 beamline at the Aichi Synchrotron Radiation Center, which is equipped with a Pilatus $100 \mathrm{~K}$ detector. Radiation energy and a wavelength used were $8.2 \mathrm{keV}$ and $1.5 \AA$, respectively. Nitrogen gas sorption analyses were carried out using the BELSORP MAX instrument (MicrotracBEL Corp.) at $77 \mathrm{~K}$. Before the measurement, the samples were out-gassed at $180{ }^{\circ} \mathrm{C}$ for $2 \mathrm{~h}$. Please see further characterization details in the ESI. $\dagger$

\section{Results and discussion}

\section{Expression of a folded lattice in carbonaceous microparticles}

For the production of the carbonaceous precipitate, an aqueous solution containing achiral, template BCP (Pluronic P123 ${ }^{\circledR}$, (ethylene oxide) ${ }_{20}(\text { propylene oxide })_{70}$ (ethylene oxide) ${ }_{20}$, PEOPPO-PEO) and D-fructose (D-Fru) was mixed and hydrothermally treated at $130{ }^{\circ} \mathrm{C}$. The scanning electron microscopy (SEM) images of the obtained powdered precipitates composed of polymeric and carbonaceous moieties show interconnected microparticles (approximately $\sim 2-4 \mu \mathrm{m}$, Fig. 1A). The particle surface shows a degree of textural orientations propagating in several individual directions (Fig. 1B, arrows indicate directions).

It must be noted that a similar hydrothermal synthesis was previously demonstrated using the same sugar but BCP with different hydrophilic chain lengths or low concentration systems. ${ }^{23,24}$ However, the framework folding has been demonstrated for the first time for the current synthesis. The absence of detailed cross-sectional investigation in the previous studies may have discounted such complex nanostructuring residing 


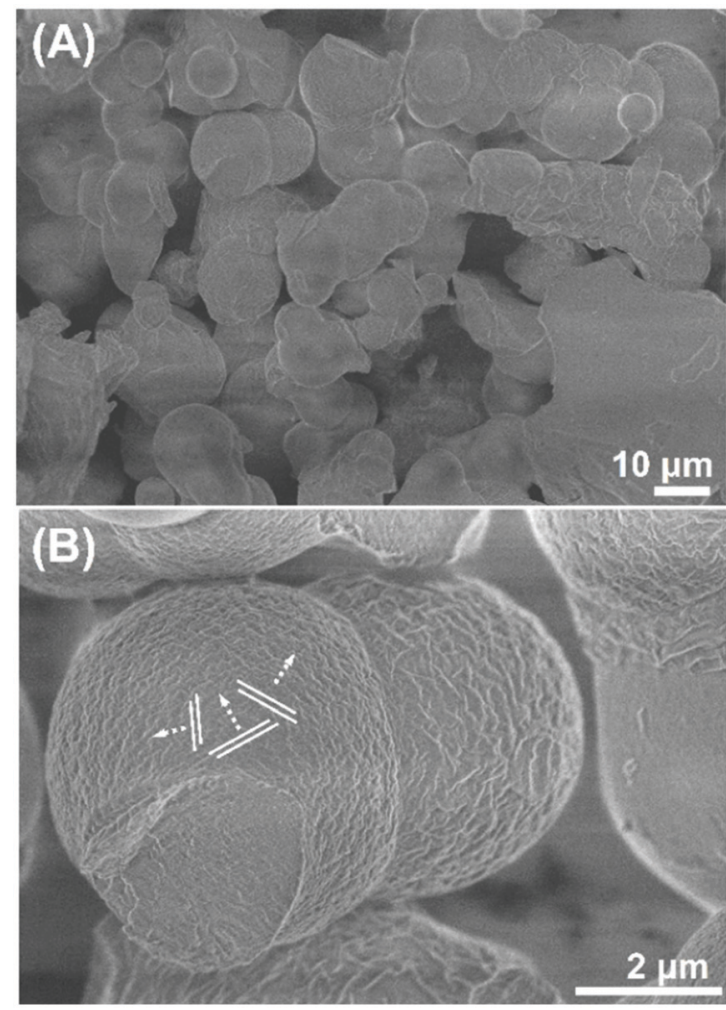

Fig. 1 (A) and (B) Scanning electron micrographs of the resulting polymer-carbon composites taken at different magnifications, with the layer developing modes indicated by lines and arrows.

inside the precipitates. Alternatively, major events responsible for the phase twisting and rotation are likely drastically enhanced in the present synthesis system. For these events to occur, the formed periodical phases must have a certain extent of bendability (elastic deformation) during the time course of the hydrothermal processing, which is perceived to be achieved in the current demonstration by creating lamellae-like, and hence, mechanically increasingly flexible,$^{25}$ that is, bendingvulnerable periodic framework compared to the previously reported systems. ${ }^{23}$ In the previously reported system, a rigid, cubic framework was formed, which was likely resistant to bending events.

The precipitates were subjected to ultramicrotome slicing for transmission electron microscopy (TEM) analysis with the achieved specimen thickness typically $\sim 50-100 \mathrm{~nm}$. Consequently, the TEM analysis provides textural information of the specimen structure in different directions at approximately the same depth, i.e., the effects due to the depth variation are omitted owing to the ultra-thin nature of the specimens. The TEM image shows the presence of lamellae-like nanostructures, while there are multiple directions of the lamellae development mode (Fig. 2A, as indicated by the sets of white lines). Individual lamellae layering modes have nearly identical repeating widths of $\sim 20 \mathrm{~nm}$ as measured from the micrograph. Careful examination of the micrograph reveals crossed lines motifs, which indicate the mutual interpenetration of the lamellae-like units. Additionally, the curved line nature suggests the existence of a twisting event occurring simultaneously with a layering event.
SSAXS analysis of the resulting composite of the polymer and carbonaceous moieties shows two main peaks at inverse nanometer $(q)=\sim 0.37$ (broad) and $0.62 \mathrm{~nm}^{-1}$ (Fig. 2B). The calculated repeating distance $(d)$ of the first peak is $\sim 17 \mathrm{~nm}$, which is comparable to the repeating distance of the lamellaelike layers observed by the TEM analysis. Notably, the $q$ value of the second peak is $1.7(=\sqrt{ } 3)$ times that of the first peak, indicating the presence of threefold symmetry in the system, where the first peak can be assigned to the (100) face of the individual lamellae units, while the second peak corresponds to the (111) face and is therefore, responsible for the threefold symmetry of a total of three lamellae units.

From the above analyses, we can establish the development of nanostructures in the synthesized carbonaceous precipitates. As illustrated in Fig. 3A-C, these nanostructures can be explained using a trefoil- or three-blade propeller-like association model. ${ }^{20}$ According to this model, there are three individual lamellae layered units, developing in individual directions, which are nearly equivalent in terms of layer periodicity, interpenetrating each other, and related to each other by a ratio of $\sqrt{ } 3: 1$, that is, a threefold axis. Each layering mode is further twisted and rotated (and can, therefore, have two comparable configurations of leftand right-handed mirror symmetries, Fig. 3A). This is further suggested by the presence of chirality in the starting sugar-BCP mixture, where $\mathrm{D}$ - and L-Fru added with $\mathrm{BCP}$ demonstrate contrastive, positive and negative CD peaks, respectively (Fig. 3B, top). In the context of molecular units introduced into the forming solid, D- and L-fructose, having hydroxy groups $(-\mathrm{OH})$ positioned in a mirror symmetry, potentially offers two contrastive hydrogen-bonded chiral configurations of BCP-sugar assemblies (Fig. 3B, bottom), i.e., via the interaction between sugar $-\mathrm{OH}$ and the PEO moiety of the lamellae forming $\mathrm{BCP}^{26,27}$ As the sum of the framework interpenetration and triple twisting events, the 3D carbonaceous nanostructure is integrated and stabilized (= folded), making up the overall particle volumes (Fig. 3C).

However, it should be noted that the threefold symmetry can be theoretically exhibited by cubic systems as well. ${ }^{28}$ Therefore, the possibility of co-formation of the cubic phase in the present system was expected and indeed a gyroid-like texture was observed locally in the system (Fig. S1, ESI†). A gyroid has a minimal surface and close thermodynamic relationship with a lamellar phase, ${ }^{29-31}$ sharing the same general folding development mechanism that produces threefold interdigitated lamellae. Hence, it would be reasonable to consider that a gyroid possesses high inclination for bending or deformation, which has been experimentally demonstrated and reported by other authors. ${ }^{32,33}$ This study does not aim to strictly distinguish the intrinsic differences between the two configurations. Instead, it considers that both belong to the family of the "layered" configurations. It may also be possible that the formed lamellar layers are oriented in a gyroidal association by surrounding the gyroidal core, thereby being hybridized and "inheriting" the gyroid's threefold symmetry, considering that the existence of such lamellar-gyroidal hybrid configuration has been reported in a real biological system. ${ }^{34}$

Nevertheless, the proposed model can account for the incidental appearance of an unusual concentric pattern observed in the 


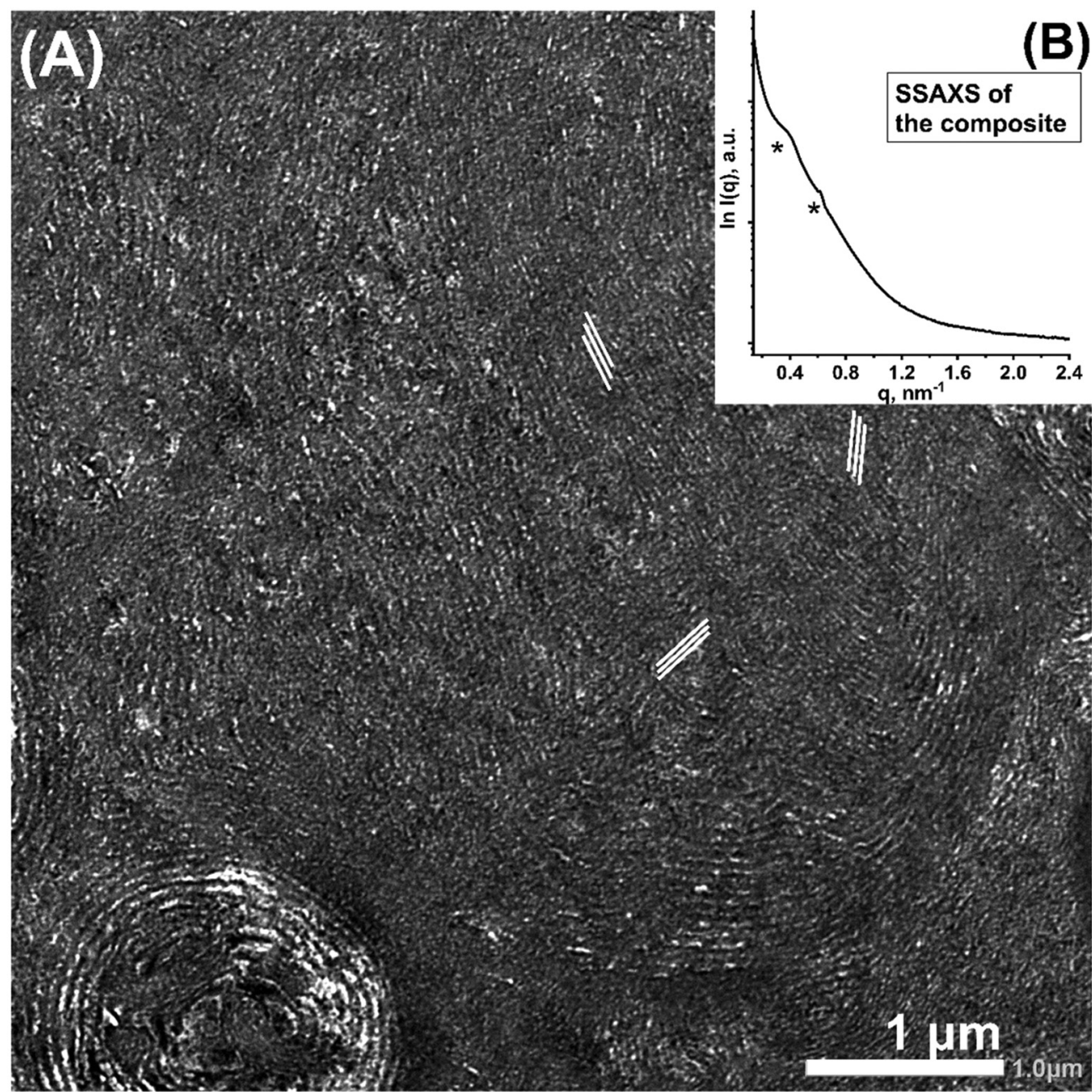

Fig. 2 (A) Transmission electron micrograph of the ultramicrotome specimen of the resulting polymer-carbon composite, showing multiple lamellaelike layers propagating in individual directions (as indicated by white lines). (B) SSAXS pattern of the resulting BCP-carbon composite.

TEM images of the ultramicrotome sliced specimen (Fig. 4A-D). Careful observation allows the detection of radially oriented textural motifs, spanning the overall particle volume (Fig. 4B-D). This detailed textural information suggests that, rather than having simple multishell structures, this particle body is made of complex superimposition of multiple mutually interpenetrating layered and deformed phases. Indeed, a careful observation indicates three layered units that individually gyrate and integrate themselves as shown in Fig. 4D (additional micrographs are provided in Fig. S2, ESI $\dagger$ ), which agrees well with the model in Fig. 3A and its approximate 2D-projected model (Fig. 4E).

The repeating distance of each lamellae-like pattern was measured to be $\sim 17 \mathrm{~nm}$, which agrees well with the periodicity as detected by SSAXS (Fig. 2B). Furthermore, focusing on the edge of the pattern, a highly interdigitated, local knot-like motif is visible (Fig. 4B). Hence, one can attribute this concentric pattern to the existence of three lamellae-like layered phases mutually interpenetrating and further, individually twisted. Specifically, the association mode may be that of a left-handed manner as characterized by the gyrating direction of the interpenetrating layers (Fig. 4D and Fig. S2, ESI $\dagger$ ) and the positional relation of the local framework association (Fig. 4B and Fig. S3, ESI $\dagger$ ).

A decaying concentric contrast pattern observed at the inner area (decaying towards the outer direction), resembling a Liesegang pattern $^{35,36}$ is potentially reflecting unique nucleation and growth modes, such as sequential precipitation events. Alternatively, the Moiré effect is considered, which is known to occur via the superimposition of several periodic or quasiperiodic patterns (of a logarithmic patterning motif), where we obtain a new pattern with new periodicities in addition to the original periodicities. ${ }^{37}$ Specifically, such a concentric decaying pattern, as observed here, has been reported to appear at the intersection of three identical phases superimposed over one another at an angular ratio of $2 \pi / 3$ (as experimentally demonstrated using three line gratings in Fig. $4 \mathrm{~F}$ ). ${ }^{37}$ If this is the case, this alternatively supports the existence of three individual ordered layered phases related by a threefold axis and the occurrence of their interpenetration.

The probability of acquiring the cross-section of the particle, cut at a plane perpendicular to the rotation axis of the three layered modules, should be limited stochastically, which might explain the very limited frequency of observing this type of 

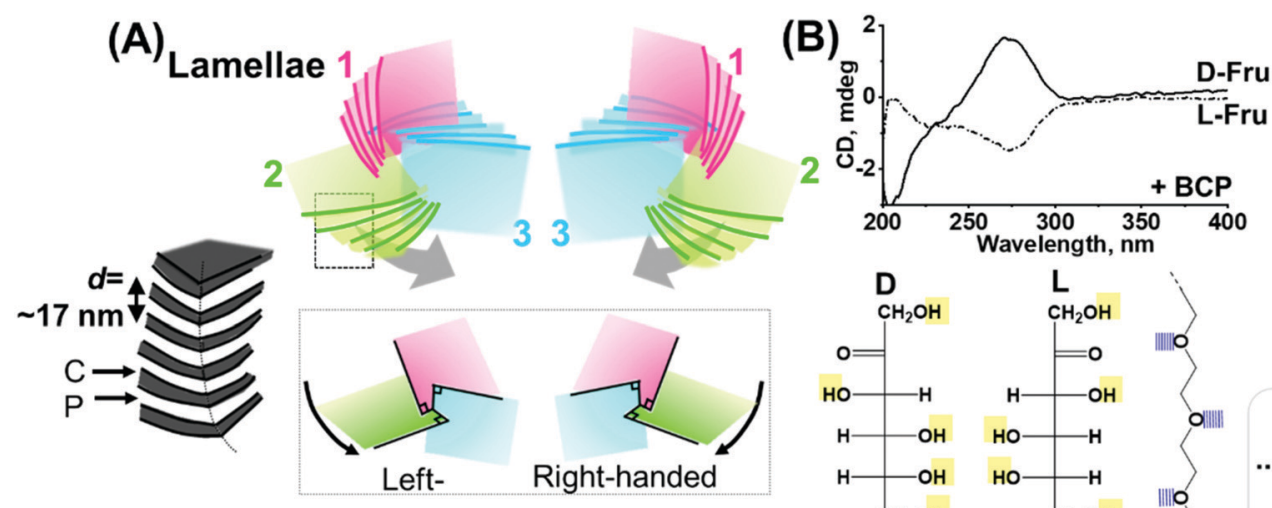

(C) $\mathrm{i}$

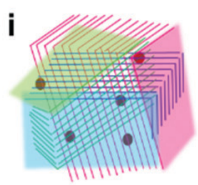

Nucleation in threefold ordered phase

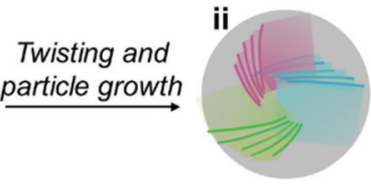

Carbon - Polymer composite

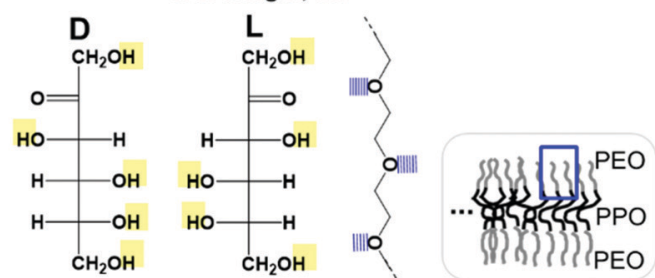

Fig. 3 (A) Proposed model of the threefold lamellae folding with left- and right-handed structural symmetries. The layer information marked in the square is depicted on the left side, with $\mathrm{C}$ and $\mathrm{P}$ corresponding to the carbonaceous and polymeric moieties, respectively (top). Local framework association mode and positional relationship of the three lamellae modules, shown by taking an equivalent single layer from each module (bottom). (B) Circular dichroism spectra of the BCP-containing D- and L-Fru aqueous solutions (top). Molecular structures of D- and L-Fru drawn using the Fischer projection and potential interaction sites with the layer-forming template BCP (= with the PEO moiety, bottom). (C) Schematic of the potential formation pathway of the folded nanostructures possessing threefold ordered lamellae-like layers.

perfect concentric pattern. Nevertheless, interestingly, in some cases, a blade-like TEM contrast motif, attributable to the (lefthanded) model shown in Fig. 3A, is observed spanning across the concentric patterns (Fig. S4, ESI $\dagger$ ). This may also reflect the existence of multiple (at least two "blades" can be identified), gyrating lamellar phases as well as the fact that the layering is accompanied by twisting and rotation events.

\section{Generation of nanopores in the folded carbonaceous framework}

The template BCP was removed by thermal decomposition (i.e., calcination at $550{ }^{\circ} \mathrm{C}$ under an inert atmosphere) to yield porous carbonaceous materials with an interdigitated nanoscale framework. These obtained carbonaceous particles demonstrated the preservation of particle size $(\sim 2-4 \mu \mathrm{m}$, Fig. $5 \mathrm{~A})$. A highermagnification image reveals a characteristic, complex surface texture similar to that observed for the composite material (Fig. S5, ESI $\dagger$ ).

The SSAXS pattern shows peak broadening. While precise peak identification is difficult, shift of the peak (or shoulder) to a higher $q$ value (approximately $0.5-0.6 \mathrm{~nm}^{-1}$ ) was observed. This shift corresponded to a repeating distance, $d=\sim 10 \mathrm{~nm}$ and structural shrinkage of $\sim 20-30 \%$ of the before-calcination structure (Fig. 5B). The peak broadening can be attributed to the enhanced twisting/rotation and associated deformation induced by additional condensation of the carbonaceous framework via calcination, which is the event that occurred at the expense of the strict ordering of the lamellae layers. However, while the hardness of the calcined particles (i.e., due to the increasingly condensed carbonaceous backbone) inhibits the preparation of the ultramicrotome specimens for TEM, a combined mechanical grinding of the calcined particles and electron microscopy analysis demonstrates the favourable preservation of ordered and folded nanostructures as well as their interpenetration modes.

The thin specimen part created by grinding of the calcined particles shows the preservation of the ordered curved lamellalike layered nature of the framework after the thermolytic template removal and their triple developing modes, indicating the preservation of the threefold configuration (Fig. 5C, see additional micrographs in Fig. S6, ESI $\dagger$ ). As mentioned for the as-synthesized composite, the coexistence of a gyroid-like nanostructure is similarly found in this calcined material (Fig. S7, ESI $\dagger$ ). Given that lamellae-like orderings cannot be generally sustained after the removal of the template without layer stabilization, ${ }^{38,39}$ preservation of the lamellae-like layering found here can be attributed to the presence of the interdigitated state of the framework (i.e., whereby resisting towards the structural collapse) and associated stabilization of the layering modes. Indeed, a careful observation of the higher-magnification micrograph (of the pointed area in Fig. 5C) indicates the presence of a complex interdigitated state in the local structure of the resulting layering framework, making a local knottinglike framework motif or a hook-like association of the framework (Fig. S8, ESI $\dagger$ ). The measured dimension of the observed periodic texture was $\sim 9 \mathrm{~nm}$, consisting of $\sim 7 \mathrm{~nm}$ of framework thickness and $\sim 2 \mathrm{~nm}$ of pore width (Fig. S8, ESI $\dagger$ ) and was found to be comparable to the approximate $d$ value obtained by SSAXS. 

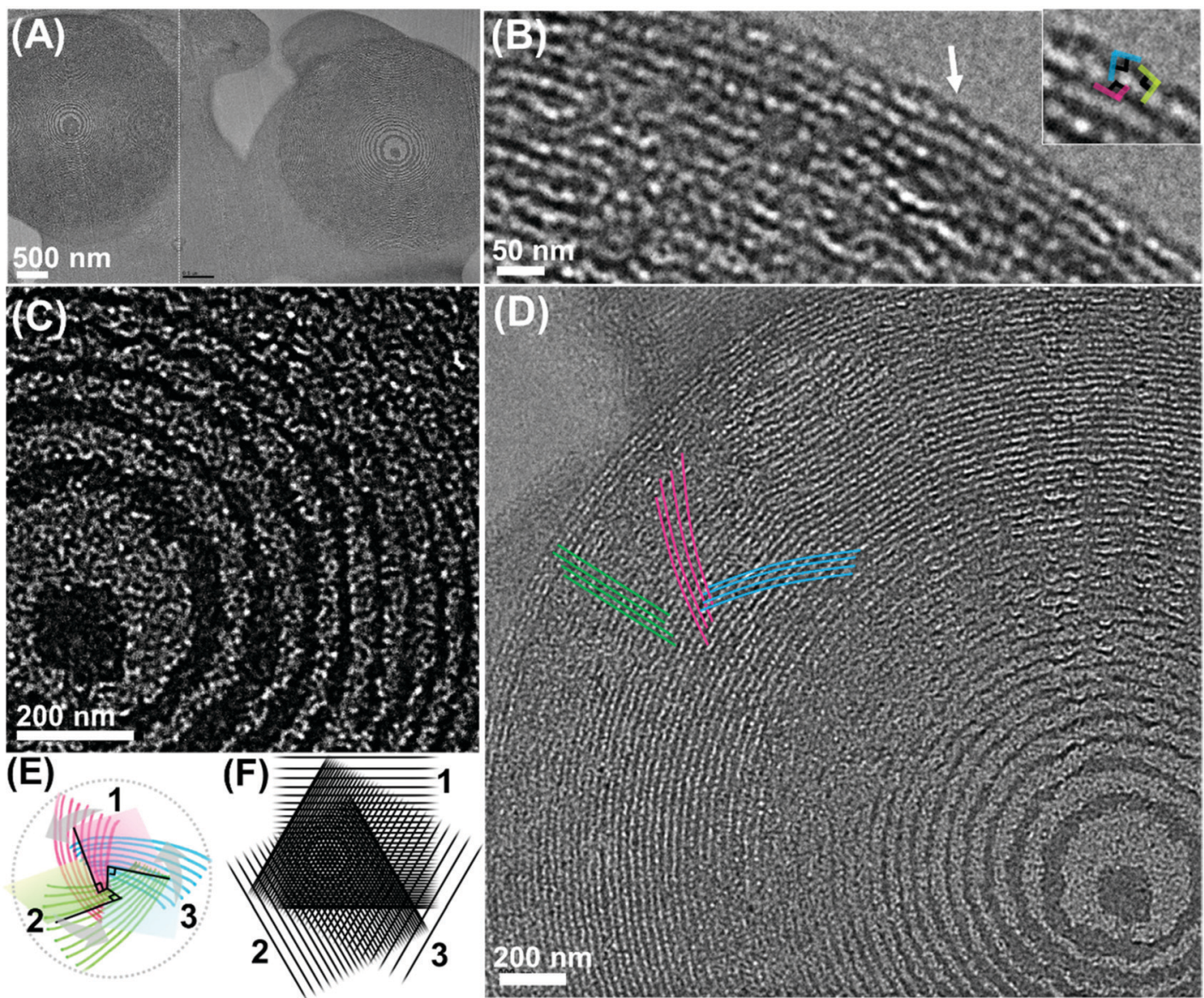

Fig. 4 (A-D) TEM images of the polymer-carbon composite showing the concentric patterns. (inset in (B)) High-magnification image of the area pointed by the arrow. (E) Illustration of the approximate two-dimensionally projected model of the configuration shown in Fig. 3A (left-handed), provided for the aiding purpose. (F) Experimental demonstration of the formation of Moiré pattern by three superimposing layers.

Notably, the particles tend to cleave at certain interfaces, particularly towards the three predetermined directions (resembling the selected cleavage tendency of planer minerals) via this mechanical grinding. This alternatively indicates the existence of three individual layering modes developed in the carbonaceous particles, and the accompanying selective cleavage in the directions parallel to the layering modes, showing a characteristic triangular particle edge shape or internal cleavage mode after grinding (Fig. S9, ESI $\dagger$ ). A left-handed twisted structure was detected upon cleaving (Fig. 5D and E), while the overall association mode i.e., via mutual interpenetration of the three layered modules, attributable to a left-handed configuration was further detected upon this cleaving (Fig. 6). From these observations, it is evident that the calcined particles favourably preserve the carbonaceous interdigitated framework of the three mutually stabilized ordered layered nanostructures.

As a result of the removal of the polymeric template, pores are created in the carbonaceous particles. Equilibrium nitrogen $\left(\mathrm{N}_{2}\right)$ adsorption experiments conducted to measure the porous properties of the material demonstrate near type I isotherms attributable to primarily microporous material characteristics (Fig. 5F). The Brunauer-Emmett-Teller (BET) surface area $\left(S_{\mathrm{BET}}\right)$ of $518 \mathrm{~m}^{2} \mathrm{~g}^{-1}$ and total pore volume $\left(V_{\text {total }}\right)$ of $0.22 \mathrm{~cm}^{3} \mathrm{~g}^{-1}$ were calculated, out of which the microporosity ( $V_{\text {micro, }}$, the volume deriving from the pores smaller than $2 \mathrm{~nm}$ ) and mesoporosity ( $V_{\text {meso }}$, that deriving from the pores larger than $2 \mathrm{~nm}$ ) were $0.18 \mathrm{~cm}^{3} \mathrm{~g}^{-1}$ and $0.04 \mathrm{~cm}^{3} \mathrm{~g}^{-1}$, respectively (Table 1 , denoted Carbon_1). The calculated pore size distribution based on the density functional theory (DFT) method was composed of a sharp peak at $\sim 0.6 \mathrm{~nm}$ in the microporous range (Fig. $5 \mathrm{G}$ ) and a less discrete small peak at $\sim 2.5 \mathrm{~nm}$ in the mesoporous range (Fig. 5G inset), while the carbon wall consists primarily of amorphous-type carbon as demonstrated by the relatively weak diffraction as analysed by X-ray diffraction measurements (Fig. S10A, ESI $\dagger$ ).

\section{Mechanistic view}

The presented nanostructure is considered to have been formed through an interplay of the chemical condensation of sugar and BCP-induced phase separation in a hydrothermal environment, which is ensured by a strong binding motif between the sugar and BCP. Specifically, hydrogen bonding between the sugar hydroxy group $(-\mathrm{OH})$ and hydrophilic PEO moiety of the template in a hydrothermal environment is considered to serve as a major nanostructuring driving force. While BCP is reported to be capable of gyrating on its own, ${ }^{40}$ such a complex yet controlled 

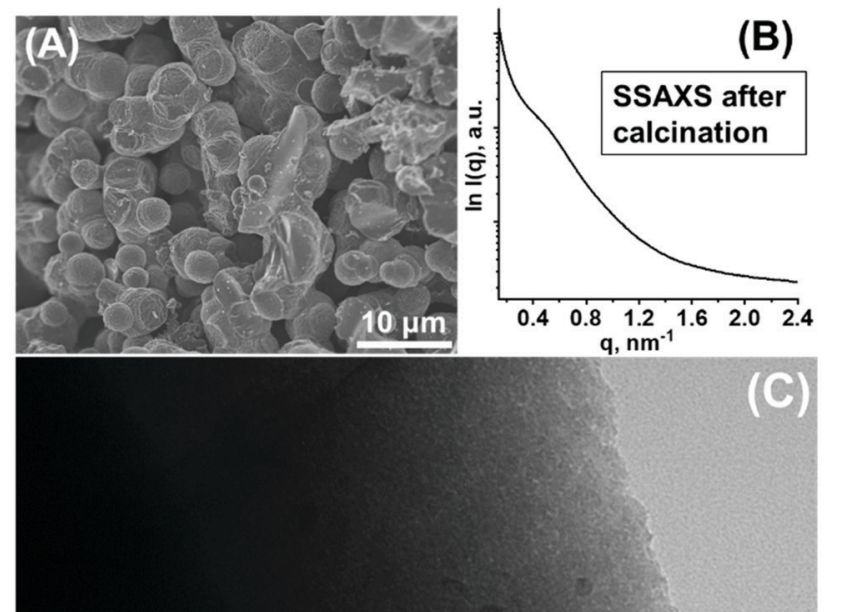

$100 \mathrm{~nm}$

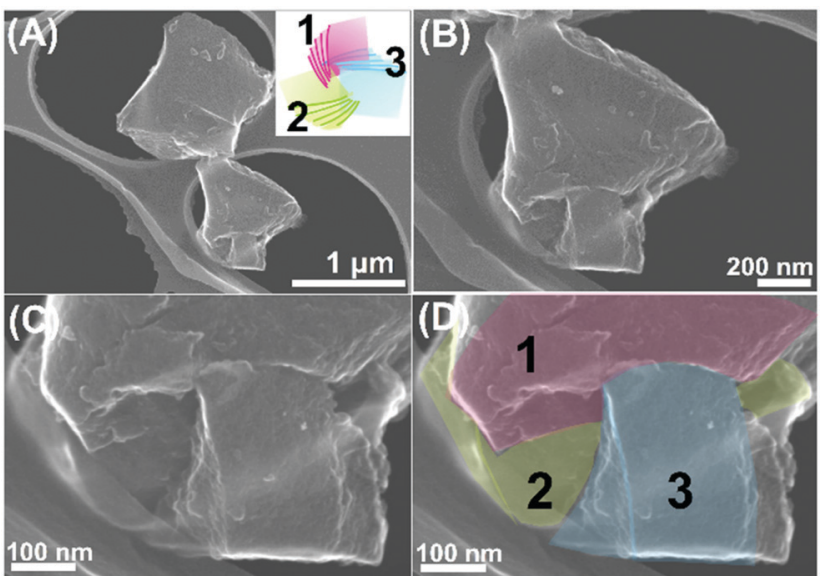

Fig. 6 (A)-(D) SEM micrographs of the carbonaceous particles after mechanical grinding (inset: the model shown in Fig. 3A). (C) and (D) show the particle in (B) at a higher magnification with the numbers of the layered modules indicated in (D).

overlay of the ordered nanoscale texture is observed only by this system, but not by other comparable carbon synthesis demonstrated using the same or similar BCP with the nonsugar carbon precursors (e.g., phenolic resins). ${ }^{39,41}$ Therefore, it can be hypothesized that the folding event is induced by Fru molecule or by Fru cooperatively with BCP, and the given structural motif is effectively "memorized" after the condensation and crosslinking are complete. To examine this hypothesis, the SSAXS measurement of the quenched gel-like precipitate at the early hydrothermal stage $(<24 \mathrm{~h})$ was performed, which demonstrated the existence of the threefold symmetry in the initial stage: the well-resolved peaks at $q=0.36-0.38$ and $0.66 \mathrm{~nm}^{-1}$ are in the ratio $1: \sqrt{ } 3$ (Fig. S11, ESI $\dagger$ ). Furthermore, an aqueous starting mixture of D-Fru and BCP generates a peak in the circular dichroism (CD) spectrum centred at $\sim 280 \mathrm{~nm}$, attributed to the left-handed optical activity of D-Fru (Fig. 3B).

Hence, in a potential scenario, interpenetration of the multiple lamellar layering modes at a threefold symmetry occurs wherein sugar is bound to the lamellae-forming BCP. Furthermore, nucleation and growth of the carbonaceous moiety occurs with accompanying dehydration and (poly)condensation of sugars, ${ }^{42}$ eventually forming a carbonaceous precipitate, where the threefold lamellar layering modes are preserved (Fig. 3C(i)). The layering modes are further twisted and rotated (i.e., folded) as the particle formation proceeds, finally to integrate themselves forming the final microparticles in an interconnected state (Fig. 3C(ii)), where the folding direction is potentially affected by the handedness of the fructose molecule.

In this context, the chiral role of the precursor sugar is considered as an interesting and new concept for hydrothermal carbon synthesis, where the relatively fast dehydration kinetics of sugar into achiral furfural moieties is well known. ${ }^{43,44}$ The presented demonstration may alternatively suggest that, under specific conditions, for instance in the presence of a chemical motif (BCP in this case) that can strongly bind sugar, sugardependent characteristics such as chirality-twisting driving 
Table 1 Pore properties of the carbonaceous materials synthesized

\begin{tabular}{|c|c|c|c|c|c|c|c|c|}
\hline Samples & $\begin{array}{l}\text { Sugar } \\
\text { type }\end{array}$ & $\begin{array}{l}\text { Template } \\
\text { system }\end{array}$ & $\begin{array}{l}\text { Calcination } \\
\text { temperature }\left[{ }^{\circ} \mathrm{C}\right]\end{array}$ & $S_{\mathrm{BET}}^{a}\left[\mathrm{~m}^{2} \mathrm{~g}^{-1}\right]$ & $V_{\text {total }}^{b}\left[\mathrm{~cm}^{3} \mathrm{~g}^{-1}\right]$ & $V_{\text {meso }}{ }^{c}\left[\mathrm{~cm}^{3} \mathrm{~g}^{-1}\right]$ & $V_{\text {micro }}{ }^{d}\left[\mathrm{~cm}^{3} \mathrm{~g}^{-1}\right]$ & $W_{\mathrm{DFT}}^{e}[\mathrm{~nm}]$ \\
\hline Carbon_1 & D-Fru & Diluted & 550 & 518 & 0.22 & 0.04 & 0.18 & $\sim 0.6$ \\
\hline Carbon_2 & L-Fru & Diluted & 550 & 534 & 0.23 & 0.04 & 0.19 & $\sim 0.6$ \\
\hline Carbon_4 & D-Fru & Concentrated & 900 & 904 & 0.41 & 0.09 & 0.32 & $\sim 0.6, \leq \sim 5.0$ \\
\hline
\end{tabular}

force-can be exerted in the system, indicating thus the specific synthetic advantage of the demonstrated hydrothermal procedure over other carbonization procedures exploited for the production of nanostructured or porous carbons. The final, selective thermal decomposition of the polymeric moieties leaves the porous carbonaceous particles with the layered frameworks interpenetrating each other in a threefold symmetric manner that are preserved to a favourable extent. The interpenetrating lamellae-like phases generate interdigitation of the carbonaceous framework and consequently the local knot-like motif (Fig. 3C(iii)).

\section{Use of enantiomeric sugars}

Assuming that sugar induces folding, stereochemical enantiomer, L-fructose (L-Fru), was alternatively used for observing potential different handedness of the nanostructural folding. The $\mathrm{CD}$ spectrum of the starting aqueous BCP-L-Fru solution gives a peak centred nearly at the same position but with negativity that can be attributed to the optical right-handedness of L-Fru (Fig. 3B). Hydrothermal treatment of the L-Fru-BCP aqueous solution yielded interconnected microparticles $(\sim 2-4 \mu \mathrm{m})$, similar to the D-Fruderived system (Fig. S12, ESI $†$ ). A highly bent and interpenetrating lamellae-like layered nanotexture was generated (Fig. 7A, arrows indicate layers). Following the electron microscopy results, the SSAXS pattern for the L-Fru-derived BCP-carbon composite exhibits similar peaks at $q=\sim 0.37 \mathrm{~nm}^{-1}$ (broad) and $0.61 \mathrm{~nm}^{-1}$ with a ratio of $1: 1.6$ between the peaks, which is close to $1: \sqrt{ } 3$ (Fig. 7B). In the calcined particles, upon mechanical grinding, a typical cleaving tendency (i.e., in three directions) and a twisted layered feature with right-handed twistedness was found (Fig. 7C and D). The interdigitation was observed at the cleaved surface of the calcined material, where, locally, a knot-like motif is found (Fig. $7 \mathrm{E}$ and F). Furthermore, overall mutual interpenetration mode of the three layered modules was detected (Fig. S13. see Fig. S10B and S14, ESI $\dagger$ for the XRD and SSAXS results of the calcined L-Fru derived material, respectively), while the material shows an $S_{\mathrm{BET}}$ of $534 \mathrm{~m}^{2} \mathrm{~g}^{-1}$ and $V_{\text {total }}$ of $0.23 \mathrm{~cm}^{3} \mathrm{~g}^{-1}$ (Fig. S15 (ESI $\dagger$ ), Table 1, denoted Carbon_2), similar microporous material characteristics as compared to the D-Fru derived carbon. In the future, determination of the selective production of the left- and right-handed chiral configurations will be pursued. Some local characteristics attributable to the nanostructural configurations with respective handedness are found in this study, which is considered to be in line with the tendency of handedness observed in the recent reports suggesting the occurrence of controlled chiral alignment for the carbonized solids synthesized from chiral precursors including sugars. ${ }^{8,21}$

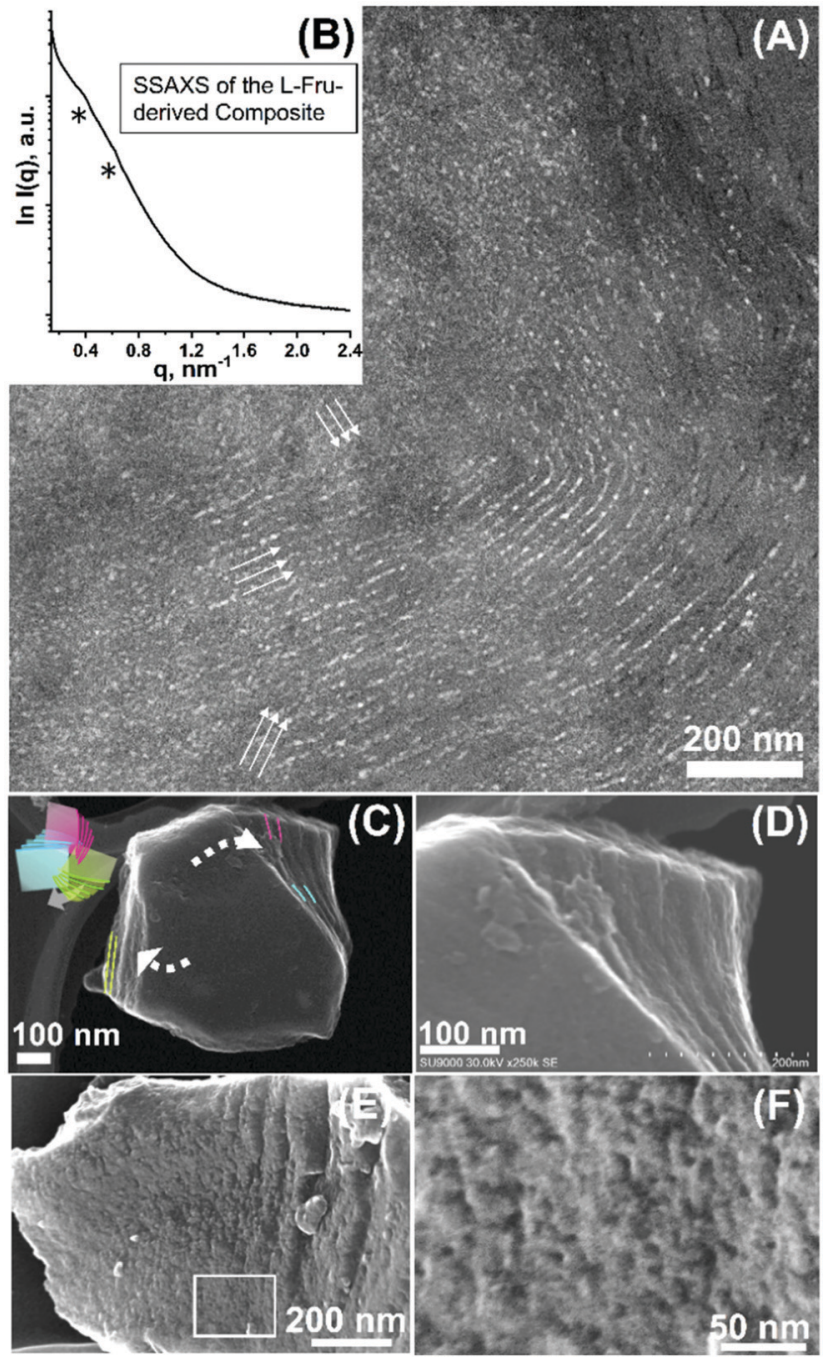

Fig. 7 (A) TEM micrograph and (B) SSAXS pattern of the carbonaceous material synthesized using L-fructose as a carbon source (arrows indicate layers). High-magnification SEM micrographs of the cleaved particle after calcination, showing ( $C$ and $D)$ twisting and ( $E$ and $F$ ) knotting-like local motifs, respectively (inset in (C): the model shown in Fig. 3A). (F) corresponds to the high-magnification image of the area squared in (E).

However, the number of examples for which handedness is examined are still very limited to refer to the degree of selectivity in this study. Furthermore, the two dimensional and nonquantitative methods impede a clear attribution of left- and right-handed folding. For instance, back projection of the lefthanded association model can appear almost indistinguishable 
to the front projection of the right-handed model. To mitigate this situation, collective and quantitative analysis of the structural handedness (e.g., via $\mathrm{CD}$ analysis of the resulting solid) or three-dimensional characterizations (e.g., via electron tomography) of the nanostructures should be performed in the future studies.

\section{Synthetic control of the nanopore dimensions}

To demonstrate that the presented approach enables a degree of variation in the nanostructural properties of the folded carbonaceous framework, synthesis was further performed at a higher polymer aqueous concentration (i.e., $\sim 3$ times higher concentration). Hydrothermal treatment of this system at

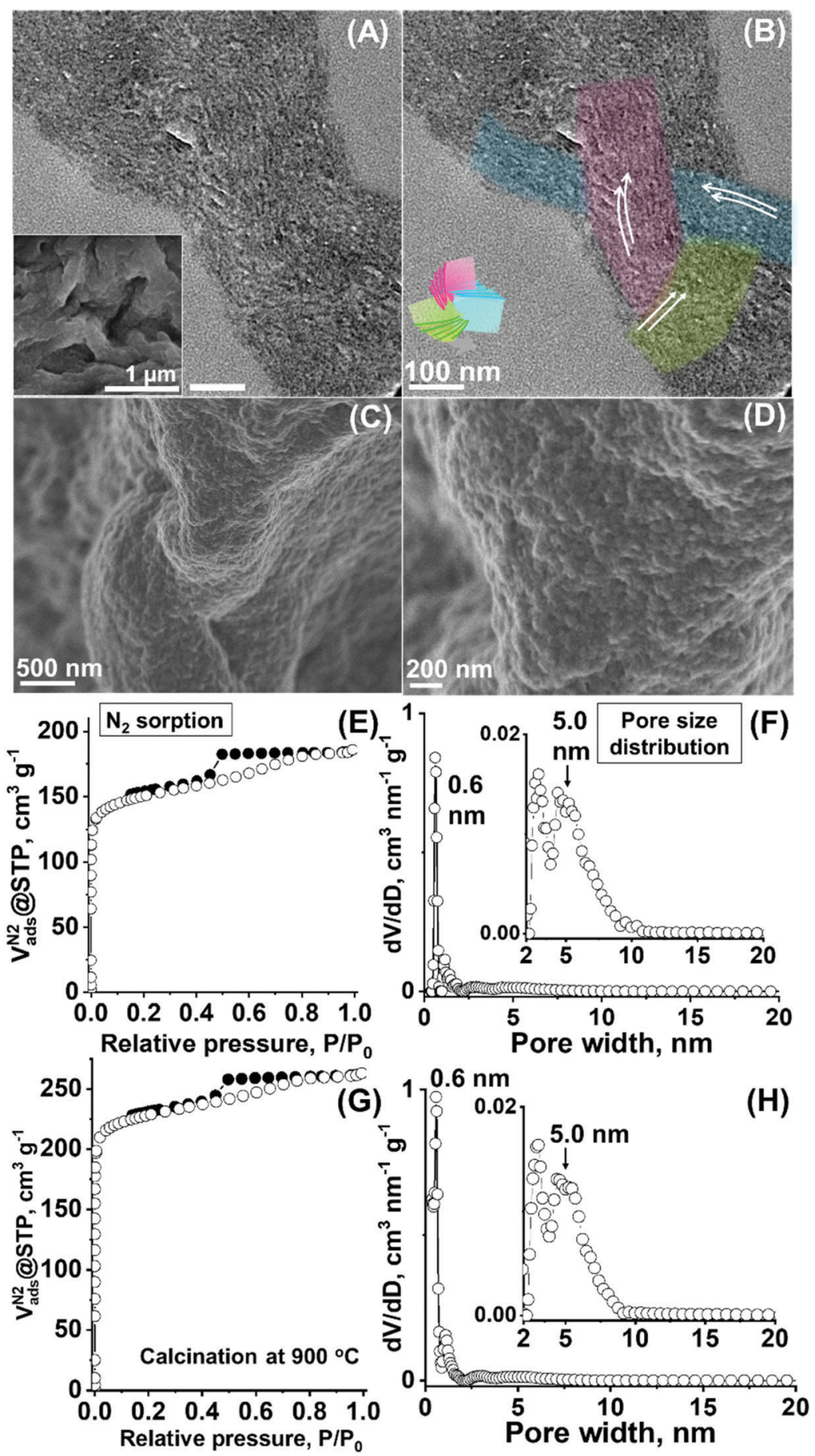

Fig. 8 (A) and (B) TEM micrographs of the composite material (inset: SEM micrograph) synthesized at a higher polymer concentration. (A) and (B) are identical micrographs, while auxiliary conductors and the model shown in Fig. 3A are added in (B). (C) and (D) SEM micrographs and (E) $N_{2}$ sorption and (F) DFT pore size distribution curve of the sample after template removal (i.e., calcination at $\left.550{ }^{\circ} \mathrm{C}\right)$. (G) $\mathrm{N}_{2}$ sorption and (H) DFT pore size distribution curve of the sample after template removal via calcination at $900{ }^{\circ} \mathrm{C}$. 
$130{ }^{\circ} \mathrm{C}$ yielded a composite of polymeric and carbonaceous moieties, while an elongated particle morphology resulted in a particle width of $\sim 200-300 \mathrm{~nm}$ (Fig. 8A, inset). This particle morphology is observed presumably as a result of the restricted growth direction of the forming carbonaceous particles due to the increased viscosity of the system. The SSAXS showed broad patterned features (Fig. S16A, ESI $\dagger$ ). While the possibility of weakening of the ordering in the sample synthesised at this concentrated template system cannot completely be discounted, this SSAXS feature is currently presumed to have resulted due to the large scattering contribution from the elongated, high-aspect ratio particle characteristics. The determination of the peak positions was difficult, however, at least the first "shoulder" peak at a slightly lower $q$ value than that for the diluted system was observed (Fig. S16A (ESI $\dagger$ ), versus $q=\sim 0.36 \mathrm{~nm}^{-1}$ and $d=$ $\sim 17 \mathrm{~nm}$ for the diluted system in Fig. $2 \mathrm{~B}$ ), attributable to the dimensional increase, while the initial quenched gel phase generated SSAXS peaks that distinctly resulted from the threefold symmetry (Fig. S17, ESI $\dagger$ ).

As an evidence of favourable preservation of the three interpenetrating layering modes in these carbonaceous particles, the TEM micrograph of ultramicrotome specimen of the particle demonstrated the presence of ordered nanostructures, i.e., three developing directions of the lamellae-like layered units (Fig. 8A and B, red, blue, and green), while the co-existence of the twisted gyroid-like textures was similarly found (Fig. S18 and $\mathrm{S} 19, \mathrm{ESI} \dagger)$. After calcination at $550{ }^{\circ} \mathrm{C}$, the particle morphology was preserved, while the pore orientation in a twisted ordered manner (Fig. 8C and D) and multiple lamellae-like and/or gyroid-like associations appeared with the twisted framework (Fig. S20 and $\mathrm{S} 21$ (ESI $\dagger$ ), see the XRD pattern for this material in Fig. S10C, $\mathrm{ESI} \dagger$ ). Similar to the diluted system, phase interpenetration was observed at the cleaved interface created by the mechanical grinding of the particles (Fig. S22, ESI $\dagger$ ), while some local knot-like motifs were also visible (Fig. S23, see Fig. S16B (ESI $\dagger$ ) for the SSAXS pattern).

Notably, larger pores are generated as a result of this variation in the synthesis protocol. A clear type IV isotherm with a capillary condensation effect, typical to the development of the mesopores, result, equivalent to $S_{\mathrm{BET}}$ of $577 \mathrm{~m}^{2} \mathrm{~g}^{-1}$ and $V_{\text {total }}$ of $0.29 \mathrm{~cm}^{3} \mathrm{~g}^{-1}$ (Fig. 8E) with the increased $V_{\text {meso }}$ value of $\sim 0.10 \mathrm{~cm}^{3} \mathrm{~g}^{-1}$ (versus $0.04 \mathrm{~cm}^{3} \mathrm{~g}^{-1}$ for the previous system, Table 1, denoted Carbon_3). An accompanying increase in the pore diameter value in the DFT distribution up to $\sim 5.0 \mathrm{~nm}$ (Fig. 8F, Table 1, with a discrete micropore peak at $\sim 0.6 \mathrm{~nm}$ ) demonstrates that the porous dimension of the folded carbonaceous material is altered. This, in turn, ascertains the effectiveness of the variation of hydrothermal synthesis parameters in controlling the pore dimension of the carbonaceous microparticles with the interdigitated and twisted nanoscale framework, thereby, demonstrating the advantage of the employed synthesis chemistry. The formed larger pores were further found to be stable up to $900{ }^{\circ} \mathrm{C}$ (i.e., via heat treatment under nitrogen), showing the maintained $V_{\text {meso }}\left(\sim 0.09 \mathrm{~cm}^{3} \mathrm{~g}^{-1}\right.$, Fig. $8 \mathrm{G}$ ) and pore size range (up to $\sim 5.0 \mathrm{~nm}$, Fig. $8 \mathrm{H}$, Table 1, denoted Carbon_4) as compared to the material calcined at a lower temperature of $550{ }^{\circ} \mathrm{C}$. The favourable pore stability for the layering mode-based ordered porous materials may be attributable to the interdigitated nature of the resulting carbonaceous framework. Furthermore, this calcination process at higher temperature develops the graphitic-like nature of the carbonaceous framework showing the pseudo-graphitic carbon wall nature, as demonstrated by the increasingly sharp peaks of the X-ray diffraction pattern of the material (peaks at $2 \theta$ of $21^{\circ}$ and $44^{\circ}$ are attributed to the (002) and (101) faces of the turbostratic-type carbon, ${ }^{45}$ Fig. S10D, ESI $\dagger$ ), which is advantageous for generating favourable electric conductivity in the porous framework.

\section{Conclusions}

The synthesis of carbonaceous particles with folding-like, longrange interdigitated and twisted ordered nanoscale frameworks has been demonstrated for the first time via the self-assembly of a sugar precursor and lamellae-forming BCP template under a hydrothermal environment. The formed nanostructure exhibits the internal threefold symmetry made of nearly equivalent lamellae-like modules, integrated by mutual interpenetration and twisting. Local framework interdigitation and local knot-like motif were also present, rendering the presented synthetic demonstration a promising basis for the construction of novel and unique solids with biological nanoscale topologies and configurations. The enhanced sustainability of the approach to yield such unique solid frameworks should further be emphasized.

Analogous to protein folding (however, unlike proteins themselves) these permanent folded frameworks made in the form of a stable condensed solid body would stimulate in-depth assessment of the geometrical attributes, such as folding, twisting, or interpenetration similar to those shown by biological assemblies, and nanoscale geometries (that are, otherwise, less accessible experimentally) along with their functions. For the applications, tunability of the pores created in the calcined carbonaceous materials were further demonstrated. The enhanced surface area, pore volume, and nanoscale pore size tunability of the produced material are significant for applications in adsorption, separation, catalysis, and electrochemistry, for instance, as nanoporous electrodes for batteries or fuel cell application studies.

While further clarification is required to elucidate the exact way of nanostructural interpenetration, including 3D configuration analysis-local angle or distance with which three layering modes are associated and the presence/degree of framework mobility), the presented unique carbon nanostructures will serve as good candidates for studying the topology-driven physicochemical properties, such as framework flexibility (e.g., swellability or durability against mechanical stress) or physical stability (due to framework integration equal to intactness) and chemical stability (reduced termini framework nature due to framework entanglement). Furthermore, studying the electron transfer phenomena (within the folded framework), mass transfer (through the tangled pore network), or photonic properties ${ }^{15,16}$ of such synthesized materials, especially in comparison with the conventional solids having noninterdigitating, clear pore network paths, would also be interesting for discovering geometry-driven transfer or light enhancement 
effects as observed in some real-life situations. Selectivity in the nanostructural handedness remains an open question; however, successful selective synthesis would allow the observation of enantiomeric separation ${ }^{8}$ or enable the study of chiral electrochemical properties of the materials.

Additionally, the current synthesis system was not free of co-precipitation of non-nanostructured spheres or aggregated bulk material. This limitation must be overcome for successful future applications, for which the control of particle growth-by minimizing possible variation/distribution of a sugar binding state, for example, by optimization of the sugar and template aqueous concentration-is considered the primary objective. Further improvement in the synthetic control will ensure the development of a facile, scalable, and sustainable approach towards the selective fabrication of high-quality materials, which would unravel interesting material properties in the above-mentioned applications.

\section{Conflicts of interest}

There are no conflicts to declare.

\section{Acknowledgements}

This work was supported by the Japan-U.S. Cooperation Project for Research and Standardization of Clean Energy Technologies, funded by the Ministry of Economy, Trade and Industry of Japan. Dr N. Kameta and Dr T. Imura are acknowledged for their support in the CD measurements. The synchrotron SAXS measurements were performed at the BL8S3 beamline of the Aichi Synchrotron Radiation Center, Aichi Science \& Technology Foundation, Aichi, Japan (Proposal number: 202002071).

\section{References}

1 P. Belmont, J.-F. Constant and M. Demeunynck, Chem. Soc. Rev., 2001, 30, 70.

2 P. Virnau, A. Mallam and S. Jackson, J. Phys.: Condens. Matter, 2011, 23, 033101.

3 A. Broom, A. C. Doxey, Y. D. Lobsanov, L. G. Berthin, D. R. Rose, P. L. Howell, B. J. McConkey and E. M. Meiering, Structure, 2012, 20, 161.

4 M. E. Evans, V. Robins and S. T. Hyde, Proc. R. Soc. A, 2015, 471, 20150254.

5 W. R. Taylor, Nature, 2000, 406, 916.

6 K. E. Shopsowitz, H. Qi, W. Y. Hamad and M. J. MacLachlan, Nature, 2010, 468, 422.

7 K. E. Shopsowitz, W. Y. Hamad and M. J. MacLachlan, Angew. Chem., Int. Ed., 2011, 50, 10991.

8 I. Fuchs, N. Fechler, M. Antonietti and Y. Mastai, Angew. Chem., Int. Ed., 2016, 55, 408.

9 P. Nuguent, Y. Belmabkhout, S. D. Burd, A. J. Cairns, R. Luebke, K. Forrest, T. Pham, S. Ma, B. Space, L. Wojtas, M. Eddaoudi and M. J. Zaworotko, Nature, 2013, 495, 80.
10 G. Prieto, J. Zečević, H. Friedrich, K. P. de Jong and P. E. de Jongh, Nat. Mater., 2013, $12,34$.

11 J. Choi, J. Kim, P. Wagner, J. Na, G. G. Wallace, D. L. Officer and Y. Yamauchi, J. Mater. Chem. A, 2020, 8, 14966.

12 S. Kubo, A. Endo and S. Yamazaki, J. Mater. Chem. A, 2018, 6, 20044.

13 S. Zhang, H. Zhang, X. Hua and S. Chen, J. Mater. Chem. A, 2015, 3, 10013.

14 S. Lee, D.-H. Kwak, S.-B. Han, Y.-W. Lee, J.-Y. Lee, I.-A. Choi, H.-S. Park, J.-Y. Park and K.-W. Park, ACS Catal., 2016, 6, 5095 .

15 J. Hielscher, C. Pouya, P. Vukusic and G. E. Schroeder-Turk, Opt. Express, 2017, 25, 5001.

16 E. Goi, B. P. Cumming and M. Gu, Adv. Opt. Mater., 2018, 6, 1800485.

17 Y. Zheng, H. Sato, P. Wu, H. J. Jeon, R. Matsuda and S. Kitagawa, Nat. Commun., 2017, 8, 100.

18 S. Che, Z. Liu, T. Ohsuna, K. Sakamoto, O. Terasaki and T. Tatsumi, Nature, 2004, 429, 281.

19 J. Maruyama, T. Shinagawa, M. Watanabe, Y. Kashiwagi, S. Maruyama, T. Nagaoka, W. Matsuda, Y. Tsutsui, S. Seki and H. Ueyama, Small, 2020, 16, 1905916.

20 X. Han, J. Zhang, J. Huang, X. Wu, D. Yuan, Y. Liu and Y. Cui, Nat. Commun., 2018, 9, 1294.

21 M.-M. Titirici and M. Antonietti, Chem. Soc. Rev., 2010, 29, 103. 22 K. Tang, R. J. White, X. Mu, M.-M. Titirici, P. A. van Aken and J. Maier, ChemSusChem, 2012, 5, 400.

23 S. Kubo, R. J. White, N. Yoshizawa, M. Antonietti and M.-M. Titirici, Chem. Mater., 2011, 23, 4882.

24 S. Kubo, Porous Carbohydrate-Derived Carbonaceous Materials, PhD thesis, The University of Potsdam, Germany, 2011.

25 Y. Iwashita and H. Tanaka, Phys. Rev. Lett., 2007, 98, 145703.

26 W. Li, C. Duan and A.-C. Shi, ACS Macro Lett., 2017, 6, 1257.

27 C. Lang, Y.-X. Shen, J. A. LaNasa, D. Ye, W. Song, T. J. Zimudzi, M. A. Hickner, E. D. Gomez, E. W. Gomez, M. Kumar and R. J. Hickey, Faraday Discuss., 2018, 209, 179.

28 A. J. Mueler, M. A. Hillmyer and F. S. Bates, Macromolecules, 2009, 42, 7221.

29 C. E. Conn, O. Ces, X. Mulet, S. Finet, R. Winter, J. M. Seddon and R. H. Templer, Phys. Rev. Lett., 2006, 92, 108102.

30 V. Saranathan, C. O. Osuji, S. G. J. Mochrie, H. Noh, S. Narayanan, A. Sandy, E. R. Dufresne and R. O. Prum, Proc. Natl. Acad. Sci. U. S. A., 2010, 107, 11676.

31 I. W. Hamley, V. Castelletto, O. O. Mykhaylyk, Z. Yang, R. P. May, K. S. Lyakhova, G. J. A. Sevink and A. V. Zvelindovsky, Langmuir, 2004, 20, 10785.

32 E. Yang, M. Leary, B. Lozanovski, D. Downing, M. Mazur, A. Sarker, A. M. Khorasani, A. Jones, T. Maconachie, S. Bateman, M. Easton, M. Qian, P. Choong and M. Brandt, Mater. Des., 2019, 184, 108165.

33 S. M. Sajadi, P. S. Owuor, S. Schara, C. F. Woellner, V. Rodrigues, R. Vajtai, J. Lou, D. S. Galvão, C. S. Tiwary and P. M. Ajayan, Adv. Mater., 2018, 30, 1704820.

34 L. Norlén and A. Al-Amoudi, J. Invest. Dermatol., 2004, 123, 715. 35 C. Rodriguez-Navarro, K. Kudłacz, Ö. Cizer and E. RuizAgudo, CrystEngComm, 2015, 17, 58. 
36 C. Rodriguez-Navarro, O. Gazalla, K. Elert and E. Sebastian, Proc. R. Soc. London, Ser. A, 2002, 458, 2261-2273.

37 O. J. Bryngdahl, J. Opt. Soc. Am., 1974, 64, 1287.

38 J.-G. Park, K. R. Lee, U.-H. Lee, H.-J. Lee and Y.-U. Kwon, J. Mater. Chem., 2011, 21, 3903.

39 Y. Meng, D. Gu, F. Zhang, Y. Shi, L. Cheng, D. Feng, Z. Wu, Z. Chen, Y. Wan, A. Stein and D. Zhao, Chem. Mater., 2006, 18, 4447.

40 S. Hezaveh, S. Samanta, G. Milano and D. Roccatano, J. Chem. Phys., 2012, 136, 124901.
41 C. D. Liang, K. L. Hong, G. A. Guiochon, J. W. Mays and S. Dai, Angew. Chem., Int. Ed., 2004, 43, 5785.

42 X. Sun and Y. Li, Angew. Chem., Int. Ed., 2004, 43, 597.

43 C. Falco, P. Caballero, F. Babonneau, C. Gervais, G. Laurent, M.-M. Titirici and N. Baccile, Langmuir, 2011, 27, 14460; C. Falco, N. Baccile and M.-M. Titirici, Green Chem., 2011, 13, 3273.

44 H. Kimura, M. Nakahara and N. Matubayasi, J. Phys. Chem. A, 2011, 115, 14013.

45 L. Yu, C. Falco, J. Weber, R. J. White, J. Y. Howe and M.-M. Titirici, Langmuir, 2012, 28, 12373. 